\title{
Thymic Basaloid Carcinoma
}

National Cancer Institute

\section{Source}

National Cancer Institute. Thymic Basaloid Carcinoma. NCI Thesaurus. Code C6456.

A rare primary thymic carcinoma, characterized by the presence of tumor cell lobules with peripheral palisading, and a basophilic staining pattern. More than half of reported cases were associated with the presence of a multilocular thymic cyst. Metastases to lung and liver have been reported in approximately $30 \%$ of cases. 